\title{
On the Integration of Verticals Through 5G Control Plane
}

\author{
Kashif Mahmood*, Toktam Mahmoodi ${ }^{\dagger}$, Riccardo Trivisonno ${ }^{\ddagger}$, \\ Anastasius Gavras ${ }^{\S}$, Dirk Trossen ", Marco Liebsch" \\ * Telenor Research, Norway, ${ }^{\dagger}$ King’s College London, UK, ${ }^{\ddagger}$ Huawei Technologies, Germany \\ $\S$ Eurescom, Germany, ๆ Interdigital, UK, || NEC Lab Europe, Germany
}

\begin{abstract}
To enable the variety of verticals and flexibly deliver services over 5G, a new control plane architecture and system concept is needed. To this end we present a vision for the 5G control plane which allows the verticals to provide tailored services over the communication infrastructure. We discuss in detail the enabling concepts which are essential to realize this vision. Finally, we present a reference framework centered around a novel control plane for vertical integration but which also takes into account the relevant ecosystem such as the underlying infrastructure, the data plane and the management and orchestration framework. We believe that the 5G control plane vision and the accompanying reference framework presented in this paper are important puzzle pieces in the overall $5 \mathrm{G}$ architecture.
\end{abstract}

\section{INTRODUCTION}

The vision in $5 \mathrm{G}$ is driven by many technological advances [1], ranging from software-driven networks over the integration of compute, storage and communication assets to the likelihood of multi-RAT access to be utilised by the significantly increased number of endpoints in the 5G world. However, the biggest change of $5 \mathrm{G}$ over the previous generations of mobile networks is the focus on the many vertical industries that together form our modern society, ranging from transportation, energy, manufacturing, media and entertainment to health [2]. It is this focus that drives many, often widely varying and even conflicting requirements into the efforts to develop novel solutions for $5 \mathrm{G}$. The variety of requirements pertains to performance, functional and architectural issues. On the performance side, while some verticals require the broader broadband $5 \mathrm{G}$ naturally aspires to, other industries consider more important low latency for small data but require large scale in the number of devices. Functional wise, some industries will aim at providing direct device to device communication while others will rely on the masterslave communication model. Finally, from an architectural perspective, innovative business will require ad-hoc network functions to tailor system behaviours to dedicated classes of devices, applications and services.

Delivering various services with such diverse requirements, needed by different industry sectors is a tremendous task, which, in today's mobile networks is to be fully handled by mobile operators. Although the type of services mobile operators offer have significantly evolved through evolution of mobile networks, their role remained the same being often the owner of mobile infrastructure, managing and maintaining the network, and running network operations. The Mobile Virtual Network Operators (MVNO) have transformed the concept of ownership, but the management and operation have remained within the sole hands of operators. With the transition to 5G, users of mobile network are expanding beyond the end users to diverse industries who are embracing the use of connectivity in running their businesses. At the same time, the role of next generation operators is moving towards empowering their future users, being enterprises or verticals, to customize, manage and even control the network associated to their own users. An example of this is enabling a car manufacturer to have its own customized AAA server for security in the control plane of the next generation communication system.

To this end, we argue that the success of 5G will highly depend on such repositioning of the operators role through enabling key concepts such as: "network slicing" and "architectural modularization", enriching resource and context awareness in service delivery, and enabling "control without ownership". In this article, we shed the light on the design principles for next generation control plane, and how this new design can transform the role of mobile operators.

The remainder of this paper is organized as follows. First, we elaborate on our vision for $5 \mathrm{G}$ control plane in Section II. To enable such vision, various technology advances are essential. Those are widely discussed in Section III where we present some major enabling concepts and finally present a reference framework in Section IV which takes into account the vision and enabling concepts previously illustrated.

\section{5G CONTROL PLANE VISION}

In addition to the many requirements posed by the specific vertical scenarios, the operator faces the constraint of providing the desired experience under an ever-increasing business dynamic, which in itself is often different in terms of speed and scale in each supported vertical industry. This requires the ability to flexibly integrate the infrastructure for various business scales that range from multi-national players to regional and local micro-industries that regulators and policy-makers alike 
are so keen to support through the future $5 \mathrm{G}$ infrastructure. All this flexibility and adaptability cannot be achieved without developing a dynamic integrated $5 \mathrm{G}$ environment, which also achieves the convergence of plethora of existing and future infrastructure, wireless and wired alike. It is therefore required (as shown in Fig. 1) to provision a perceived integrated infrastructure which accommodates the requirements defined by the verticals, while utilizing the mobile and fixed infrastructure as well as digital assets which can be owned by multiple stakeholders or even by the verticals themselves. It needs to be emphasized here that the term "perceived" refers to the ability of $5 \mathrm{G}$ to hide to the customers the actual complexity and heterogeneity (in terms of technology domains included and ownership) of the physical and virtual infrastructures over which the tailored communication systems are deployed and operated.

\section{A. Key Design Principles}

A key design principle towards $5 \mathrm{G}$ control plane is architectural flexibility, i.e. the capability to adapt to the different verticals. This also includes flexibility in service deployment, so that we can create services faster and in a more costefficient way. A second design principle is the openness of the control plane for service creation, which is a must when moving towards the goal of being a digital service provider. Thirdly, the 5G control plane must provide connectivity via a multitude of access technologies and not being limited to cellular radio only. Finally, the control plane and the network functions therein should support context awareness by design to enable smart decision processes and optimized operation at functional level.

\section{B. Major Constraints}

Apart from the specific requirements posed by each of those vertical industries, control plane for $5 \mathrm{G}$ also faces general constraints as defined by the expected $5 \mathrm{G}$ ecosystem (shown earlier in Fig. 1, namely (i) sustainable TCO, (ii) business dynamicity, (iii) business scalability and (iv) business compliance. While (i) is known from existing generations of mobile network, albeit complicated by the ever increasing number of vertical industries expected to make use of the infrastructure at likely ever increasing cost pressures, the other three items are more clearly driven by the expected transition from a largely mobile broadband-oriented mobile industry as of today to a $5 \mathrm{G}$ world in which the communication infrastructure is expected to serve as the nervous system of the Digital Society across a large number of vertical industries.

\section{ENABLING CONCEPTS}

In addition to the well-recognized advances in softwarized systems, most prominently software-defined networking (SDN) [3] and network function virtualization (NFV), we believe that any solution for an integrated 5G infrastructure and communication system must realize the following concepts.

\section{A. Network Slicing}

The concept of Network Slicing, which is also now agreed in 3GPP [4] is expected to become the key feature of next generation networks, providing the flexibility required to support the variety of 5G use cases and business. Network slices are seen as network operator business, offering the possibility to provide flexible services and even infrastructures to vertical industries and classical Telco customers alike. In such a scenario, each slice is tailored to different functional and performance requirements, potentially operated isolated from each another but instantiated and running on a common virtual and/or physical infrastructure. So a network slice is an independent logical network defined by the interconnection of a subset of required network functions (NFs) (as shown in Fig. 2). It needs to be emphasized that the network slice is defined across the whole communication system, hence including both access and core network functions and may involve last hop connection resources, including spectrum, radio resources, processing and storage [5].

\section{B. Smart Connectivity}

Wide consensus exists around the expectation that $5 \mathrm{G}$ shall provide architecture and performance flexibility, and network slicing is one of the candidate principles towards integration of vertical industries. However, it is indeed unclear which different slices will be required, and how such slices will differ from each other. The consensus on this so far does not provide a spin for further innovation in $5 \mathrm{G}$ architecture design. This aspect is also reflected in the current status of relevant standardisation work. There are currently very few agreed features which will enable a quite moderate slice differentiation: e.g. referring to 3GPP SA2 work [4]: the introduction of mobility levels, or QoS Framework for Resource Management in Mission Critical group communication. One of the show stoppers in the evolution of $5 \mathrm{G}$ architecture is in fact the connectivity model. Using the current connectivity model, i.e. the EPS bearer model, control plane only provides data plane a means to transfer data from a device to an external PDN gateway. Variations of this connectivity model may include connection oriented or connectionless schemes, or may relate to the support of e.g. customised QoS, mobility, security models.

A novel connectivity model can be one of the main enablers of vertical integration in 5G network. New Smart Connectivity models should enable a communication, computation and storage (CCS) graph, interconnecting multiple logical entities, potentially relating to multiple owners. In this case, the network data plane can build up a complex interconnection graph (CCS graph), which provides inter-connectivity among a set of different devices. The CCS graph can then also include multi-endpoints communication schemes and enable multiconnectivity and access to multiple slice from a single device, by an inherit design. 


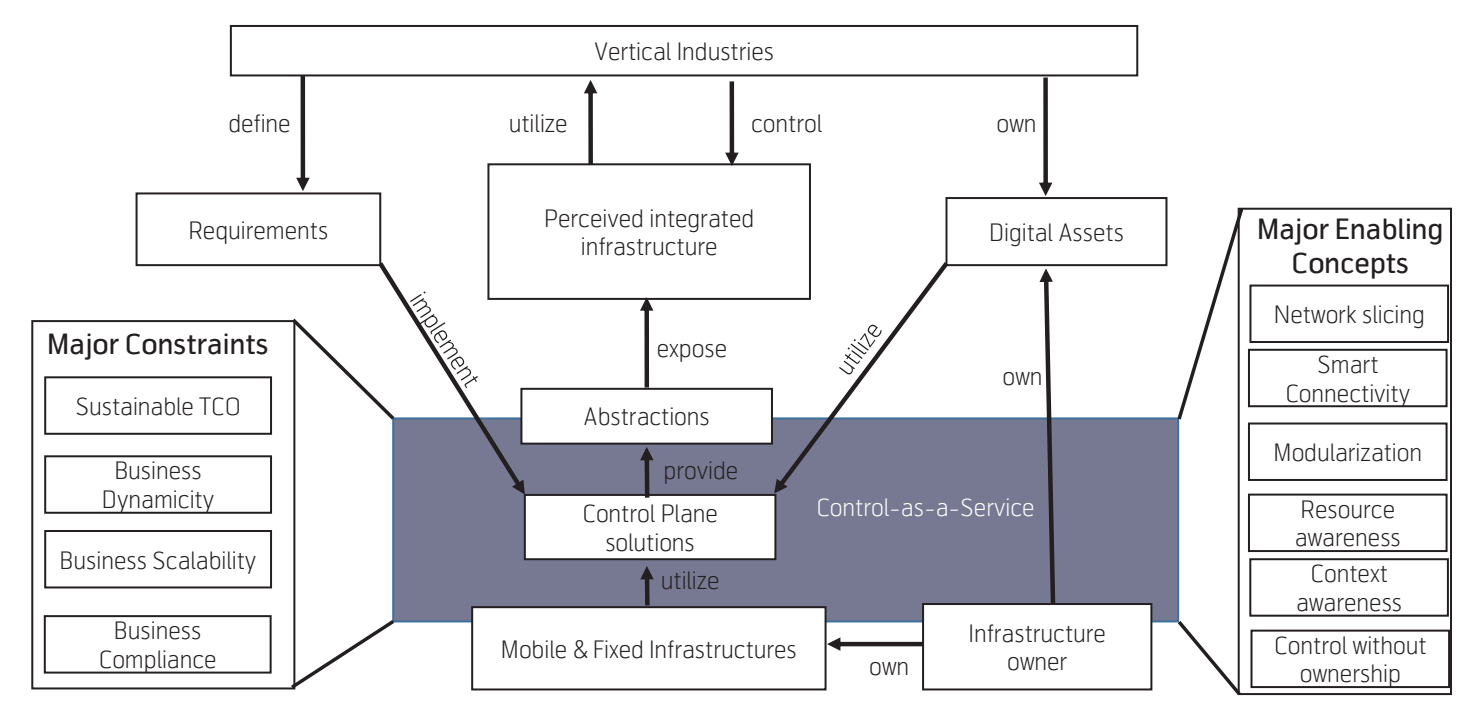

Fig. 1. A functional representation of $5 \mathrm{G}$ system with the focus on control plane and depicting major constraints and enabling concepts

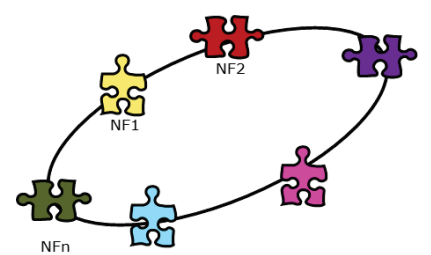

Fig. 2. A Network slice is an interconnection of a subset of required Network functions

\section{Architecture Modularization}

While slicing provides the desired isolation to enable multiple scenarios, the major concept of architecture modularization provides next generation networks with the flexibility required by the diverse set of $5 \mathrm{G}$ use cases defined by the vertical business, and for a native support of network slicing. Furthermore, architecture modularization is also a mean towards heterogeneous access networks integration. The architecture modularization concept is regarded as an essential design principle to build a flexible network architecture natively supporting network slicing. According to this concept, conventional monolithic Network Functions (NFs) (often corresponding to physical network elements in the existing systems) and new functionality envisaged for $5 \mathrm{G}$, are to be arranged into new modular NFs defined with the proper granularity, allowing the definition of different logical architectures [6] which ultimately correspond to different networks slices.

\section{Resource Awareness}

It is well recognized that $5 \mathrm{G}$ KPIs will not be purely achieved through faster 'pipes' and more spectrum. Instead, services will need to be realized along all dimensions of connectivity, computation and storage. Therefore, awareness of resources in the dimensions of computation, storage and communication must be achieved at the level of the end- to-end (E2E) network architecture. A holistic approach to awareness and control of such resources across the entire E2E network fabric is the key to realize mobile edge computing and fog computing visions in order to stem the data deluge that is inevitable with the proliferation of many billions of digital assets (e.g. in the form of IoT devices being connected to a $5 \mathrm{G}$ system), together with the expected explosion of ever-immersive services that will produce and consume many times more audio-visual content than is being transported over today's network.

\section{E. Context Awareness}

Resource awareness has also to be complemented by context awareness [7], to streamline and magnify the user experience. For instance, aspects such as determining the best connectivity choice, in terms of interface or point of attachment to the network, for a set of terminals, taking into consideration their capabilities, the requirements of the service they are using, the conditions of the link, and even the preferences of the users themselves, allows for an optimization of the user experience, as well as of the resources and network operations necessary to provide that experience. Context awareness for optimizing control plane and data plane has gained a lot of interest in the recent years [8]. For example, the 3GPP SA2 working group which is listing the key issues for next generation mobile networks has highlighted context awareness as one of the decisive feature to be supported. However, there are a number of challenges related to its effective adoption.

- The contexts used in today's 4G network, such as Packet Data Network (PDN) subscription context, Mobility Management (MM) context, and Evolved Packet System (EPS) bearer context are not rich enough to allow for efficient optimisation. For instance, the PDN context includes the identity of a PDN gateway and Access Point Name (APN), indicating which gateway and APN are used, 
while no information regarding the PDN subscription usage and status is provided.

- In today's network, context is handled in a nonconsolidated way, which means different NFs process and handle context data locally, and as a consequence, reusability is extremely complex. A related challenge deals with the ability to quantify the gain from context usage for different network procedures, such as policy enforcement procedure.

- The single context ontology would be sufficient only if $5 \mathrm{G}$ network operates in one specific environment. On the contrary, 5G needs to be customisable according to the requirements and highly varying conditions in which vertical services might operate. Hence, there is a need to accommodate the likely differences in vertical industries' ontologies.

- Given that different wireless technologies operate in a substantially different manner and need specific aspects for allowing their optimised usage, an appropriate convergence is necessary to exploit access technology contextual information as input for optimised networking utilisation.

To this end there is a need to develop a context aware framework which is able to:

- Generate rich context, taking into account diverse information such as the heterogeneous access technology contextual information.

- Support data fusion mechanisms, such as filtering, transformation, rate of change estimation.

- Handle any context ontology, while allowing for a spatial and temporal decoupling of fact and context knowledge; in this way the knowledge may evolve over time at both the concept and fact levels to meet the changes in the supported use cases.

- Enable novel concepts in context derivation, such as selflearning of context.

\section{F. Control Without Ownership}

The support of network slicing over an integrated infrastructure also brings into the spot the need of clarifying the control - ownership pair, thus generating the novel concept of control without ownership. In such a scenario, a new stakeholder can offer control plane as a service to a client, say a railway company having a radio network with masts along the rail tracks. This will be useful for scenarios where the client may own the network infrastructure but does not necessarily have the knowledge and expertise to manage it.

\section{Reference Model \& Framework}

In this section, we present a reference model that shows how the above discussed enabling concepts can be brought together and deliver $5 \mathrm{G}$ control plane addressing integration of verticals in the next generation communication system. This reference model, depicted in Fig. 3, includes 5G control plane and data planes, both physical and virtual infrastructures, resource controllers, NFV management and orchestration (MANO) platform, and 5G OSS/BSS systems.
The logical control and data planes are separately represented as red and green rectangles respectively in line with the Control and User Plane Separation (CUPS) principle. The parallel red rectangles are indicated to represent the existence of different control planes (made by different sets of control plane network functions), customized for different network slices. The modularized control plane architecture consists of Control plane Network Functions (C-NFs) that are represented by the red puzzle pieces where the different shades of red capture the different type of C-NFs. The light red puzzle pieces represent the NFs owned by the control plane stakeholder. Examples of C-NFs are mobility management, flow management, connectivity management and context awareness NFs. A detailed description of these C-NFs can be found in [6].

Context awareness NF is used by the other NFs for context aware decision making. For example connectivity management NF can use context information related to the application for anchor point reselection. A detailed description of context awareness framework and its applications can be found in [8]. The core C-NFs are interconnected via BBi interfaces, while core and access C-NFs are interconnected via WBi interfaces.

The dark red puzzle piece represents the possibility to integrate control plane functions provided by verticals or $3 \mathrm{rd}$ parties within the network slice. Such functions are connected to C-NFs via north bound interfaces (NBi). An example of such a case could be for e.g. an automotive manufacture wants to have its own customized flow management NF. So the 5G control plane should be able to integrate an external NF. It is also important to highlight here the red chequered puzzle piece which indicates the NF responsible for handling resource awareness with respect to the control plane. The two different placements of this NF highlight the possibility of this NF to be either in-house or externalized. An analogous description to the above applies to the data plane where we have data plane network functions (D-NFs) represented by the shaded green puzzle pieces.

The modular 5G control and data planes of different network slices are instantiated over the physical/virtual infrastructure which are represented in the blue rectangles in the lower part of Fig. 3. This includes access, transport, computational and storage resources which are separately represented. Parallel blue rectangles are depicted to represent the existence of multiple tenants for physical/virtual infrastructures. The physi$\mathrm{cal} /$ virtual infrastructures are controlled by resource controllers which are connected to physical/virtual infrastructures via resource specific interfaces (CRi_X). The modular network functions which run on this infrastructure could be implemented, either as virtual network functions, which connect to resource controllers via southbound interface $\mathrm{SBi}$, or as control applications running over resource controllers (e.g. as control application running on SDN controllers), or even as dedicated hardware equipment.

For the sake of completeness, Fig. 3 also includes the OSS/BSS systems (yellow rectangle) allowing possibly network slice management as well and E2E network slice orchestration. Finally, on the right hand side of the picture, the 


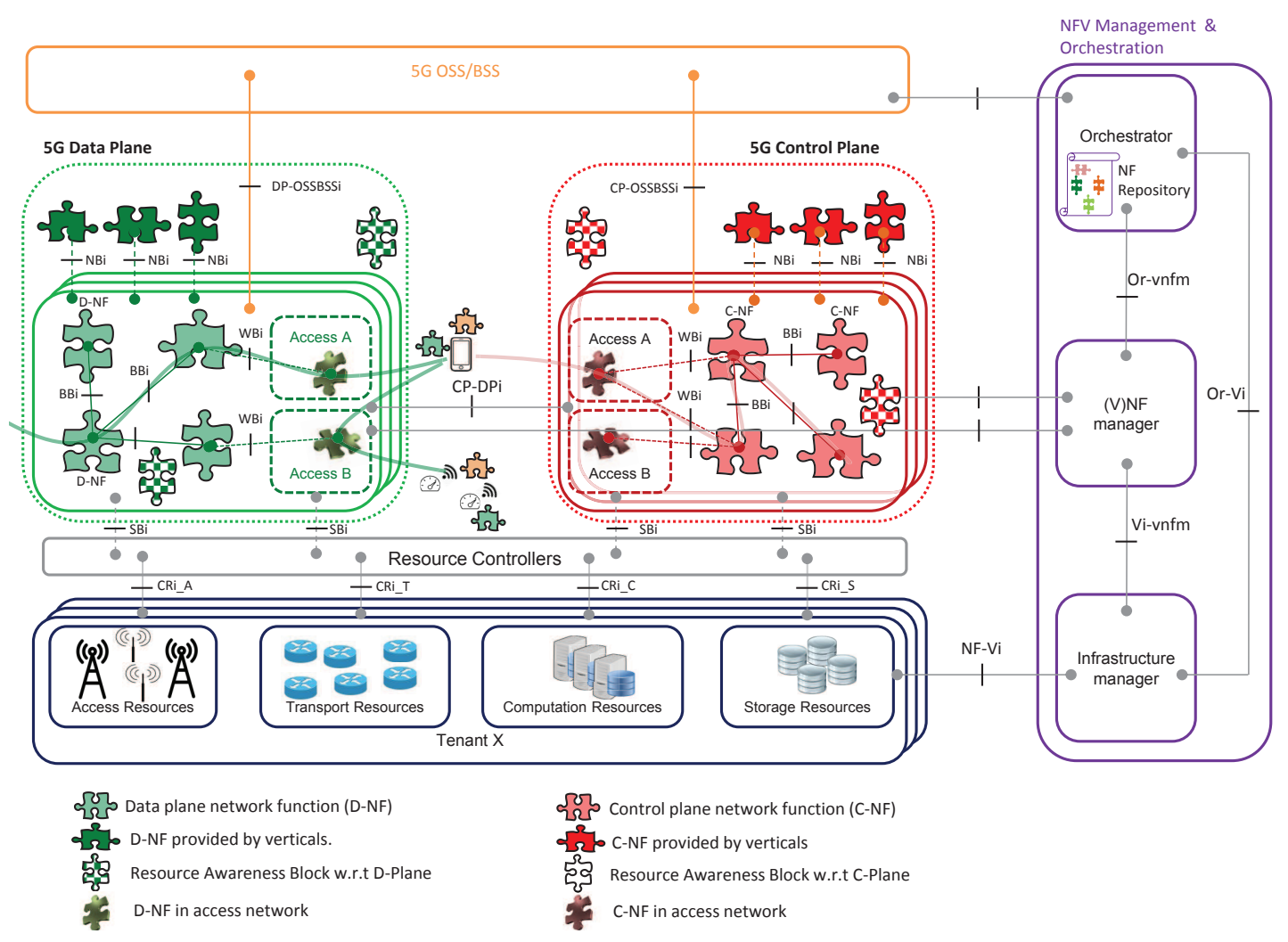

Fig. 3. Reference Model with vertical centric control plane and highlighting the key enabling concepts.

standard ETSI NFV MANO platform is illustrated along with the relevant reference points, which allows NFV infrastructure orchestration across different virtual infrastructure managers, service lifecycle management, VNFs lifecycle management, and virtualized infrastructure management.

The reference model and framework presented in this paper is a first attempt towards a control plane which allows vertical integration while incorporating the different enabling concepts.

\section{Conclusion And Future Directions}

The role of a next generation operator is that of delivering a tailored communication systems to its customers (end users, enterprises or verticals) and, even more challenging, of enabling its customers (enterprises or verticals in this case) to customize, manage and even control the communication systems. Such shift can not be achieved unless some fundamental aspect of the network architecture are redesigned. Focusing on the key evolution aspect of 5G in integrating vertical industries, this paper elaborates the enabling concept and framework through which those concepts could be developed.

\section{ACKNOWLEDGEMENT}

The authors would like to thank Rui Aguiar, Hans Einsiedler, Xiaofeng Huang, Tarik Taleb, Slawomir Kuklinski, Xueli An, Daniel Corujo, Filipe Leito, Panagiotis Gouvas an Damien Lavaux for the fruitful discussion and brainstorming in shaping the concept and ideas detailed in this paper.

\section{REFERENCES}

[1] A. Gupta and R. K. Jha, "A survey of 5G network: Architecture and emerging technologies," IEEE Access, vol. 3, pp. 1206-1232, July 2015.

[2] 5GPPP, "5G Empowering Verical Industries-," Tech. Rep., February 2016.

[3] T. Mahmoodi and S. Seetharaman, "Traffic Jam: Handling the Increasing Volume of Mobile Data Traffic," IEEE Vehicular Technology Magazine, vol. 9, no. 3, pp. 56-62, September 2014.

[4] "Study on architecture for next generation system (release 14), v14.0.0," 3GPP, Release 14, 2016.

[5] M. Jiang, M. Condoluci, and T. Mahmoodi, "Network Slicing Management and Prioritization in 5G Mobile Systems," in European Wireless, May 2016.

[6] X. An et al., "End-to-end architecture modularisation and slicing for next generation networks," CoRR, vol. abs/1611.00566, 2016. [Online]. Available: http://arxiv.org/abs/1611.00566

[7] S. Nunna, A. Kousaridas, M. Ibrahim, M. Dillinger, C. Thuemmler, H. Feussner, and A. Schneider, "Enabling Real-Time Context-Aware Collaboration through 5G and Mobile Edge Computing," in 12th International Conference on Information Technology - New Generations, April 2015, pp. 601-605.

[8] C. C. Marquezan et al., "Context awareness in next generation of mobile core networks," CoRR, vol. abs/1611.05353, 2016. [Online]. Available: http://arxiv.org/abs/1611.05353 